\title{
Tangence
}

\section{La lecture du fait divers : fonctionnement textuel et effets cognitifs}

\section{Christian Vandendorpe}

Numéro 37, septembre 1992

Autopsie du fait divers

URI : https://id.erudit.org/iderudit/025725ar

DOI : https://doi.org/10.7202/025725ar

Aller au sommaire du numéro

Éditeur(s)

Tangence

ISSN

1189-4563 (imprimé)

1710-0305 (numérique)

Découvrir la revue

Citer cet article

Vandendorpe, C. (1992). La lecture du fait divers : fonctionnement textuel et effets cognitifs. Tangence, (37), 56-69. https://doi.org/10.7202/025725ar d'utilisation que vous pouvez consulter en ligne.

https://apropos.erudit.org/fr/usagers/politique-dutilisation/ 


\section{La lecture du fait divers: fonctionnement textuel et effets cognitifs}

Christian Vandendorpe *

Le fait divers n'a pas bonne presse, si l'on peut se permettre ce jeu de mots. Sa lecture laisse facilement soupçonner chez celui ou celle qui s'y adonne une certaine complaisance pour l'horreur, qui viendrait d'une curiosité "malsaine " ou d'un obscur besoin de revanche sur la vie. Mais, en même temps, ces histoires "vraies * se vendent bien, voire de mieux en mieux, et certains journaux ne vivent que de cela.

Au lieu de céder à la réprobation qui pèse sur le genre tout entier et à l'horreur que nous inspire en particulier le texte étudié dans ce numéro («Une famille massacrée à la hache "), nous le questionnerons pour les informations qu'il peut nous apporter sur les mécanismes de lecture et ce qu'ils impliquent, tant sur le plan mental que textuel. Cela constitue une façon, parmi d'autres, de mettre l'horreur à distance et de nous faire échapper, du moins pour un temps, à la paraphrase qui risquerait ici de s'épuiser en une litanie impuissante.

\section{La lecture des scripts}

Lire un fait divers, même aussi simple que celui-ci, oblige à mobiliser des connaissances de divers types. Car le sens n'est pas donné d'emblée et sa construction exige que nous sachions effectuer les liens avec des savoirs multiples et parfois très spécifiques. Cette constatation me semble constituer un acquis relativement récent, que l'émergence du paradigme cognitif a banalisé. Un analyste aussi subtil que Roland Barthes, à qui l'on doit une remarquable réflexion sur le fait divers, pouvait encore écrire, en 1962:

le fait divers [...] est une information totale, ou plus exactement, immanente; il contient en soi tout son savoir: point besoin de connaître rien du monde pour consommer un fait divers; il ne

* Université d’Ottawa. 
renvoie formellement à rien d'autre qu'à lui-même [...] Au niveau de la lecture, tout est donné dans un fait divers; ses circonstances, ses causes, son passé, son issue; sans durée et sans contexte, il constitue un être immédiat, total, qui ne renvoie, du moins formellement, à rien d'implicite. 1

Cet immanentisme triomphal ne me semble pas pouvoir résister à une lecture attentive de ce fait divers.

Certes, il est exact que l'essentiel de la nouvelle se comprend en soi : un adolescent abat sa famille à la hache. Voilà un contenu probablement transculturel, chaque être humain possédant les catégories (Venfant/, /meurtre/, /famille/) qui lui permettent de penser l'événement et d'en percevoir le tragique. De ce point de vue, il est donc vrai que cette histoire est complète. Elle présente, de par la nature même des acteurs qui y sont impliqués, une péripétie au sens aristotélicien du terme, soit sle retournement de l'action en sens contraire. ${ }^{2}$ et elle possède assez d'étendue quoiqu'on se situe probablement ici à la limite inférieure en deçà de laquelle l'effet de récit s'abolirait - pour montrer *le passage du bonheur au malheur, toujours selon les termes de l'auteur de la Poétique. Bonheur dans les visages rayonnants de cette famille américaine typique, telle qu'elle apparait sur la photo; horreur du massacre suggéré par le texte.

Mais nombre de notations du texte seraient incompréhensibles pour qui n'aurait pas une connaissance intime de la société américaine. Cela apparaît dès la deuxième phrase: "Le procureur a fait savoir qu'il ferait en sorte que le garçon soit jugé comme un adulte et non comme un mineur. Que vient faire dans le récit cet acteur qu'est le procureur? Pour être correctement décodée, cette information suppose tout d'abord une connaissance, au moins sommaire, du fonctionnement de la justice, à savoir, premièrement, qu'un personnage est chargé de poursuivre, au nom de l'État, l'auteur d'un méfait et de requérir une peine contre lui. Et deuxièmement, qu'il existe deux procédures possibles de jugement, celle qui s'applique aux mineurs, catégorie dans laquelle devrait normalement entrer un adolescent de 16 ans, et celle qui s'applique aux adultes. Mais ces connaissances objectives ne sont toujours pas suffisantes pour permettre une lecture correcte de

1 Roland Barthes, "Structure du fait divers", Essais critiques, Paris, Seuil, 1964, p. 189.

2 Aristole, Poétique, 1452a 24. 
l'information donnée par cette phrase. Il faut encore pouvoir en cerner les implications pragmatiques, ainsi qu'on le verra plus loin.

Une certaine connaissance du monde est nécessaire aussi pour interpréter correctement la phrase suivante:

Le garçon, qui s'était en partie rasé la tête et qui avait teint le reste de ses cheveux en noir avant que les cadavres ne soient découverts, était en cours d'interrogatoire à la poste centrale de Rochester, une ville de 60000 habitants.

La plupart des lecteurs auront probablement corrigé automatiquement la faute de genre (la poste) et supposé que l'interrogatoire se déroulait bien au poste de police et non au bureau de poste: on imagine mal les policiers procédant à leur interrogatoire parmi les sacs de courrier et les clients venus acheter des timbres ou percevoir un mandat-poste!

Mais cette même phrase contient une notation bien plus énigmatique, à savoir que "le garçon [...] s'était en partie rasé la tête et [...] avait teint le reste de ses cheveaux en noir *. D'où vient ce rituel barbare? Quel signe le jeune homme veut-il donner par cet acte? Pour qui est au fait des réalités américaines de cette fin de siècle, le crâne rasé et la teinture des cheveux évoquent le script du punk: incarnation d'une révolte adolescente extrême contre le monde des adultes, sur fond culturel de drogue et de violence. Le style punk est une sécrétion d'un mode de vie extrêmement normé et conventionnel, tout comme le mythe de l'artiste romantique s'est construit par antithèse avec le monde bourgeois. En adoptant ce style, l'adolescent se pose en rupture radicale avec l'image rassurante de la photo, à l'opposé de cette vie conformiste que ses parents pouvaient lui donner en exemple, dans leur maison de Cascade Township en banlieue de Rochester.

La conjonction du crâne à demi rasé et du meurtre violent n'est pas sans évoquer aussi la scène culminante du film Taxi Driver, de Martin Scorsese (1982). On y voit un chauffeur de taxi - incarné par Robert de Niro - qui, poussé par une déconvenue amoureuse, décide de passer à l'action violente contre une bande de proxénètes, faute d'avoir pu s'en prendre à un politicien en campagne présidentielle. Lorsqu'il part pour sa mission, bardé d'armes diverses, celui qu'on connaissait jusque-là comme un paisible chauffeur de taxi s'est coiffé à la punk, tel que le sera le garçon de notre fait divers. Était-il son modèle direct et le fait 
divers n'est-il qu'un avatar *intertextuel * de Taxi Driver? Ou tous les deux renvoient-ils au modèle plus large du punk, symbole de révolte adolescente bien ancré dans la société américaine? Le texte ne nous permet malheureusement pas de trancher.

Ainsi cette nouvelle prend-elle davantage sens et épaisseur de réalité à partir du moment où l'on parvient à identifier les scripts dont relèvent certaines des actions rapportées. Défini par Schank et Abelson comme un schéma cognitif correspondant à une séquence d'événements récurrents ${ }^{3}$, le script nous fournit un contexte à l'aide duquel une information donnée est réinterprétable non pas comme la création pure et simple d'un individu, mais comme la reprise et la modulation d'un fait culturel ou social préexistant. On pourrait dire, en quelque sorte, que le script est à une action ce que le phénomène de l'intertextualité est à une citation ou à une allusion: la seconde renvoie au premier, dont elle apparaît comme un avatar ou une reprise. C'est le script qui nous permet d'interpréter une donnée comme significative, tant il est vrai que le sens ne vient jamais que des liens que nous sommes à même d'établir entre deux éléments. Parce qu'ils contiennent notre savoir socio-culturel, les scripts nous permettent de retracer les liens du sens là où l'auteur du texte les avait laissés dans l'ombre, les tenant sans doute pour acquis chez son public lecteur.

À cet égard, la dernière phrase du récit est également lourde d'implicite culturel: «Un écolier nous a dit qu'il avait des problèmes avec son père en raison d'une cassette qu'il avait achetée et qu'il ne voulait pas que son père écoute. . Cette donnée, qui semble le mobile du crime, ne peut être comprise, elle aussi, que par un lecteur sensible au rôle de la musique dans la culture des jeunes Américains. Depuis les années soixante, la musique s'est taillé un auditoire chez les adolescents comme elle n'en avait jamais eu auparavant, ce qui a contribué à développer une nouvelle culture, avec ses codes et ses valeurs propres. Elle détrônait ainsi la culture du livre par où venait traditionnellement l'idée d'un comportement marginal, qu'il s'agisse d'un Julien Sorel auquel son père reprochait d'être un *lisard *, ou d'une Madame Bovary qui s'était étourdie dans la lecture de romans sentimentaux. La nouvelle culture s'affirme dans nombre de chansons qui

3 R. Schank et R. Abelson, Scripts, plans, goals and understanding, Hillsdale, Erlbaum, 1977. 
60

prennent à contrepied la culture ambiante, telle qu'elle est représentée par les adultes, les parents. Au jeu de la transgression, certains groupes rock - je pense au beavy metal - sont parfois allés très loin, exaltant la violence, le suicide, jouant avec un imaginaire démoniaque... Cela a d'ailleurs suscité aux États-Unis un nouveau débat sur la censure et des groupes rock ont même été poursuivis en responsabilité par des parents dont un enfant se serait suicidé sur l'influence de leurs chansons.

L'adolescent de notre récit aurait donc eu en sa possession une cassette dont les paroles exacerbaient le fossé qu'il pouvait y avoir entre lui et son père. C'est le souci de ne pas en dévoiler le contenu, de protéger sa culture propre, qui aurait constitué le mobile du massacre. Mobile mais non motif. Si le mobile est d'ordre rétrospectif, le motif est plutôt d'ordre prospectif ${ }^{4}$ et porte sur les bénéfices escomptés. Quels seraient-ils ici? Peut-être l'adolescent envisageait-il de vivre selon l'esthétique punk, comme l'indique le fait qu'il se soit en partie rasé la tête et teint les cheveux immédiatement après son forfait. À ce stade de l'analyse, on ne peut qu'être saisi d'un retour de l'horreur éprouvée à la première lecture: est-il possible qu'un motif aussi vague ait pu justifier une telle frénésie de meurtre?

Ce qui fait l'efficacité du fait divers tient à la grande disponibilité des scripts qu'il met en jeu. Il n'a besoin que d'énoncer l'essentiel: le reste se déduit de la connaissance que nous avons du monde représenté, ce qui réduit au minimum les notations d'ordre descriptif. En ce sens, le fait divers met à nu les implicites culturels d'une société. Corrélativement, plus une société est différente de la nôtre, plus ses faits divers devront être "racontés", "expliqués. pour être pleinement compréhensibles. Mais les faits divers de type monstrueux qui envahissent nos journaux sont le plus souvent d'origine locale et n'ont pas besoin d'être médiatisés par l'écriture, ni contextualisés: ils sont immédiatement recevables, avec toute leur charge de proximité.

De ce point de vue, le fait divers est aux antipodes de la science-fiction, qui exige de la part du lecteur une capacité de décentrement culturel et d'imaginaire que tous ne possèdent pas.

4 Pour un rappel de la distinction classique entre mobile et motif, voir Bertrand Gervais, Récits et actions, Longueuil, Le Préambule, coll. -L'Univers des discours *, 1990, p. 96-97. 
Il exploite les schèmes correspondant à notre univers quotidien. Il recueille ce qui, dans la banalité ambiante, est élevé à la dimension du tragique ou du cocasse 5 . Aussi reste-t-il facilement compréhensible quand bien même les informations en sont faiblement connectées, bourrées d'ellipses, et l'organisation peu cohérente.

\section{Une textualité réduite}

La construction du texte mérite qu'on s'y arrête. Le * fait divers - désignant à la fois l'événement rapporté et le type de texte qui le rapporte, le syntagme est doublement connoté. Comme texte, parce qu'il rapporte, selon la définition du Lexis (1979) * des nouvelles peu importantes intéressant une personne ou un groupe restreint de personnes ; comme événement, parce qu'il est lié à des comportements hors norme, qui suscitent en nous des émotions archaïques, dont la plus fondamentale est peut-être la peur. Mais la dépréciation dont souffre le genre tient peut-être aussi à ses conditions de production et à la forme de textualité qui le caractérise. Cela apparaîtra à l'examen de la construction de notre fait divers.

L'article comporte cinq paragraphes, dont le premier est en caractères gras. La fonction de ce paragraphe d'introduction, aussi appelé . chapeau ", est de capter l'attention du lecteur, tout en lui fournissant l'essentiel de l'information. Comme le note Philippe Gaillard: *Le lecteur doit recevoir des premières lignes le maximum possible d'information. S'il s'intéresse au sujet, il poursuivra sa lecture; sinon il sera quand même mis au courant. " 6

Quand on y regarde de plus près, cette règle du journalisme implique une double exigence, à savoir résumer l'information qui va suivre et donner au lecteur le désir d'en savoir plus. Certaines histoires s'y prêtent bien, tel ce modèle proposé par Harold Evans

5 J'ai montré ailleurs que les faits divers pouvaient être classés en deux grandes catégories, le cocasse et le monstrueux, selon la place qu'ils font a la dimension cognitive, et que c'est la première surtout qui se prête à un rapprochement avec la structure de la fable. Voir mon essai De la fable au fait divers, Montréal, CIADEST, Cahier de recherche $\mathrm{n}^{\circ} 10$, 1991.

6 Philippe Gaillard, Tecbnique du joumalisme, Paris, PUF, coll. .Que saisje? =, 1971, p. 87. 
62

dans son manuel: "Chicago, Oct. 31 : James Wilson lighted a cigarette while bathing bis feet in benzine. He may live. 7 Comme le fait remarquer Evans, "Eugene Doane's intro is a rare piece. It manages to tell the whole story from beginning to end." Et il est vrai que le lecteur, tout en étant renseigné sur les tenants et les aboutissants de cette histoire, voudra savoir pourquoi le pauvre homme se baignait les pieds dans de l'essence, et d'où lui est venue la malencontreuse idée d'allumer une cigarette. Le fait de savoir déjà l'essentiel n'ôtera probablement pas l'envie de lire toute l'histoire. De même, l'enfant aime-t-il se faire re-conter une histoire, tout comme on aime se rejouer au ralenti une scène qui nous a étonné: c'est ce qu'exprimait déjà Virgile dans son vers fameux "Bis repetita placent.

Mais dans bien des cas, la règle relative au chapeau introductif peut fausser la cohérence du texte. C'est ce qui se passe ici, du fait que le rédacteur a cru nécessaire de placer dans le chapeau une phrase relative aux intentions du procureur. Certes, on a ainsi d'entrée de jeu les tenants et aboutissants de l'histoire, mais cela produit un résumé éclaté, intégrant à la mention du meurtre les modalités de sa sanction, qui apparaissent dérisoires et fort secondaires en regard du crime.

En plus de rompre avec le thème de la phrase qui précède, cette précision sur les intentions du procureur crée un problème d'enchaînement avec le deuxième paragraphe, où l'adolescent est replacé en position thématique. La déclaration du procureur revient dans le troisième paragraphe, sur le mode direct cette fois. Une telle insistance sur le fait que l'adolescent va être jugé comme un adulte, qui occupe près de vingt pour cent du texte, est a priori assez étonnante et fera l'objet d'une analyse ultérieure.

C'est seulement dans le quatrième paragraphe que sont donnés des détails relatifs au meurtre, en répondant aux questions classiques du qui, du où et du comment. Le cinquième, enfin, évoque un mobile possible, le pourquoi, et nous apprend que l'adolescent aurait parlé à ses amis après le crime, soit dans la journée du jeudi. On ne sait comment interpréter la phrase -L'un d'entre eux nous a dit qu'il avait des problèmes avec ses parents». Faut-il la comprendre comme renvoyant à la période

Newsman's English, London, Heinemann, 1972, p. 84. 
qui a précédé ou suivi le quadruple assassinat? Dans cette dernière hypothèse, nous serions en présence d'un euphémisme assez remarquable.

Le texte se clôt sur une dernière citation, qui tombe un peu à plat, comme si les ciseaux du metteur en page étaient passés par là. Peut-être avons-nous tout simplement ici un exemple de la structure dite de la "pyramide inversée * qui caractérise la composition de bien des faits divers. Le procédé est ainsi décrit par Philippe Gaillard:

À cela s'ajoute l'image de la pyramide inversée qui commande de placer les éléments dans un ordre décroissant d'importance. Là encore il s'agit de faciliter le travail du secrétaire au marbre qui pourra, en cas de besoin, couper les derniers alinéas sans avoir besoin de chercher à travers tout l'article les passages les moins importants. Il s'agit aussi d'aider le lecteur et de ne le priver, s'il abandonne sa lecture avant la fin, que d'éléments secondaires. ${ }^{8}$

Ainsi le journaliste de faits divers doit-il rédiger son texte comme si celui-ci allait être coupé! Quand on sait combien un texte forme normalement une totalité où il est aussi difficile d'ajouter que de retrancher une phrase, on peut apprécier l'effet dévastateur de cette double contrainte imposée par une certaine conception du journalisme: rédiger un texte qui soit complet (et donc avec une conclusion) tout en prévoyant que les derniers paragraphes pourraient être coupés. Cette règle étant assez régulièrement suivie par la grande presse américaine, on comprend d'où vient l'impression, souvent éprouvée à la lecture de cette dernière, que plus on avance dans un texte, plus est forte l'impression de redondance, les paragraphes de la fin ne faisant que reprendre sous une forme délayée des informations déjà livrées dans le corps de l'article: le journaliste, prévoyant la chute du couperet, ne se soucie plus de donner une conclusion à son texte, mais enchaîne simplement des paragraphes à contenu informatif de plus en plus faible. Dans l'article qui nous occupe, nous échappons à ces redites, sans qu'il soit bien évident si cela est dû à une coupure faite lors du montage dans Le journal de Montréal ou à une autre étape de la mise en forme de cette nouvelle car celle-ci provient, comme on sait, d'une agence de presse américaine et a donc dû être traduite. Toujours est-il qu'il 
64

manque à la lecture ce sentiment de totalité signifiante que l'on tire normalement de la conclusion d'un texte.

En somme, tout comme on a vu que le fait divers nous fournit en négatif des informations sur une société donnée, de même peut-on dire qu'il fonctionne souvent comme le négatif d'un bon texte. Ce qu'on nous donne a lire, ce sont des bribes d'information recueillies par des journalistes pressés, mises en texte ou agglutinées par des scripteurs anonymes, traduites à la hâte et montées dans un journal pour des lecteurs également pressés. Les aspects rhétoriques sont à peu près nuls. L'objectif n'est pas de faire jouer le langage, mais de rapporter le fait brut, celui-ci étant déjà bien assez remarquable en soi pour susciter l'émotion du lecteur.

Mais ne sommes-nous pas trop sévères? Pouvons-nous attendre du journaliste qu'il donne une conclusion à ce qui n'est encore qu'un fragment d'une nouvelle en train de se faire, d'une histoire en train de se construire? L'interrogatoire de l'adolescent vient seulement de commencer, après quoi viendra l'instruction, puis le procès. Ce n'est qu'à ce moment - ou parfois même bien plus tard - que l'on pourra véritablement raconter cette histoire, en faire une nouvelle, un roman, voire une tragédie. Un Stendhal se penchera alors sur les mobiles qui poussèrent Julien Sorel à tirer sur sa maîtresse, un Flaubert se demandera comment Madame Bovary a pu finir si lamentablement, ou un Truman Capote retracera l'itinéraire de Dick et Perry, ces criminels qui planifièrent de sang-froid l'exécution sauvage d'une famille du Kansas.

\section{Un discours de l'immédiat}

En attendant, le journaliste, qui est bien forcé d'écrire en fonction des matériaux fort incomplets qu'il a en main, relatera les événements en employant les temps du passé en conjonction avec ce temps particulier qu'est le passé composé - que Benveniste appelle le temps du discours ${ }^{9}$ - par opposition au passé simple. Le passé composé est en effet le temps du passé toujours en train de se faire, auquel n'a encore été assignée nulle ligne d'horizon d'où l'on pourrait raconter une histoire en toute objectivité et en l'embrassant dans sa totalité. Il est le temps d'un récit

9 -Les relations de temps dans le verbe français *, Problèmes de linguistique générale, 1, Paris, Gallimard, coll. .Tel •, 1966, p. 237-250. 
qui ne peut ou ne veut s'avouer tel, et dont on ne connaîtra que plus tard toutes les composantes.

Cet emploi du passé composé s'accompagne tout naturellement de déictiques qui renvoient à la situation d'énonciation. On apprend ainsi dès la première phrase que l'adolescent a été arrêté . hier . Comme le journal est daté du 20 février 1988, ce qui, selon mon calendrier électronique, coïncidait avec un samedi, on peut reconstituer la chaîne des événements de la façon suivante: le massacre a eu lieu le jeudi matin, l'adolescent s'est en partie rasé la tête et teint les cheveux avant que les cadavres ne soient découverts (quand?), il a parlé avec ses amis dans la journée de jeudi et a été arrêté par la police le vendredi. (Nous supposons ici que la nouvelle a été publiée par Le journal de Montréal dès qu'elle lui est parvenue sur les téléscripteurs, ce qui est loin d'être sûr, car, dans certains journaux, les faits divers sont utilisés comme bouche-trous, sans respect pour la vérité référentielle des événements qu'ils relatent.) Comme l'indique l'imparfait duratif -L'adolescent [...] était en cours d'interrogatoire ", la nouvelle a été rédigée alors que le criminel était toujours interrogé par la police, soit probablement le vendredi. Il faut en conclure que le déictique "hier " est ici régi par l'instance de lecture, instance que le rédacteur place anticipativement dans la journée du samedi, et non par celle de l'écriture. La fameuse subjectivité qui se manifeste dans le discours par les déictiques ne serait donc pas toujours celle que l'on croit, mais résulte parfois du jeu croisé de celle du rédacteur avec celle du lecteur.

\section{Comprendre à ses risques et périls}

Tout comme l'énonciateur projette sa subjectivité (ou celle qu'il attribue au lecteur) dans le langage, le lecteur reçoit ce qu'il lit à partir de sa propre subjectivité, en le faisant passer, comme on l'a déjà indiqué, à travers les filtres de ses connaissances, de ses expériences, de ses schèmes cognitifs. Or, les filtres les plus profonds sont sans doute ceux qui touchent à l'intégrité physique. C'est ce qui explique la puissance des émotions que peut provoquer la lecture de ce fait divers et qui nous force à abandonner la notion d'un sens toujours neutre et qui serait purement intellectuel. Loin de pouvoir se réduire à une abstraction chimiquement pure, le sens apparaît ici comme fortement imprégné de composantes émotives. 
À la dixième ou douzième lecture de ce fait divers, je ne peux m'empêcher d'être encore bouleversé par la représentation de ce qui s'est passé. Ainsi qu'on l'a vu plus haut, la compréhension immédiate de la nouvelle exigeait que je fasse les liens entre les événements, que je relie le comportement de l'adolescent aux faits énoncés dans les divers paragraphes. Pour cela, il m'avait fallu procéder à un travail actif d'inférences, afin de replacer les événements dans l'ordre chronologique, identifier correctement le lieu de l'interrogatoire comme étant le poste de police, établir un lien entre l'écoute d'une certaine cassette et l'état d'esprit du jeune homme, entre la coiffure qu'il a adoptée après le crime et la culture punk, etc. Ce sont ces inférences qui permettent d'établir correctement les liens entre les phrases, mais aussi de combler les lacunes du discours. Une fois le processus de représentation amorcé, il suffit d'un rien de concentration pour apercevoir plus finement les divers enchaînements d'actions impliqués par les données disponibles: le mûrissement de la décision, l'horreur de la scène, la peur et les cris des victimes. Je me projette ainsi le plus facilement du monde dans la situation de cette famille réveillée dans l'aube blafarde d'un matin d'hiver pour trouver son destin sous la hache d'un adolescent déchaîné. A partir des bribes que me fournit l'agence de presse, c'est une totalité agissante que je reconstruis, une séquence temporelle continue. Ce faisant, je me suis introjecté les schèmes de l'assassin aussi bien que la terreur de ses victimes: j'ai recréé les structures mentales qui permettent de penser un tel forfait.

On voit ici que, sous l'angle du sens, on ne peut effectuer de partage rigide et constant entre le rationnel et l'émotif. Pour Marvin Minsky ${ }^{10}$, *nos émotions les plus fondamentales sont des processus internes au moyen desquels des schèmes mentaux proto-spécialisés contrôlent ce qui se passe dans notre cerveau * (p. 172). Pour lui, *tenter de distinguer entre les structures émotionnelles et intellectuelles d'un adulte revient tout simplement à décrire les mêmes structures à partir d'un point de vue différent * (p. 328. Notre traduction). Nos émotions sont d'autant plus fortes qu'elles mettent en jeu des processus d'autoconservation ou réactivent des structures très anciennes. Ainsi, selon le même auteur, attribue-t-on au cri d'un bébé le degré d'urgence qu'on

10 Marvin Minsky, The Society of Mind, New York, Simon and Schuster, 1986. 
aurait soi-même dû éprouver pour crier avec la même intensité (p. 171).

Comprendre quelque chose, c'est toujours nécessairement être partie prenante et activer ou créer en soi les schèmes qui nous en permettent la représentation ${ }^{11}$. Aussi doit-on développer des moyens de tenir à distance le monstrueux, en faisant comme si cela ne nous concernait pas. Comment calmer l'appréhension qu'un tel événement pourrait nous arriver à nous aussi, que nous pourrions à notre tour être la victime d'un comportement monstrueux? Comment conjurer une telle violence? Comment prévenir l'apparition d'un tel comportement chez nos propres enfants?

La mise à distance la plus spontanée, la plus courante, est celle qui provient d'une réaction axiologique. La condamnation morale de l'acte monstrueux permet alors de rétablir l'équilibre à un autre niveau. Elle garantit, dans une certaine mesure, que les schèmes que nous nous étions forgés pour comprendre l'acte en question seront dûment étiquetés comme non acceptables moralement et qu'ils ne devront donc pas être repris à notre compte ni intégrés dans notre répertoire actif. C'est ce que traduit l'axiome populaire selon lequel "le crime ne paie pas.

C'est en ce sens qu'il faut sans doute interpréter l'insistance du texte sur la décision du procureur de faire juger l'adolescent comme un adulte. L'horreur du crime est telle qu'elle ne peut être compensée et tenue à distance que par l'idée d'un châtiment exemplaire. Aussi cette déclaration doit-elle être intégrée à la nouvelle - dont elle constitue le point d'orgue accompagner le résumé du crime dans le chapeau introductif. En arrivant de façon précipitée, au moment où on annonce que le criminel (présumé) vient d'être arrêté, elle donne la mesure du sentiment d'horreur et d'insécurité qui devait alors régner dans la population de Rochester. Par sa déclaration, le procureur annonce un retour à la norme et prévient les émules potentiels du jeune homme qu'une telle transgression de l'ordre social ${ }^{12}$ ne

11 Montaigne, qui portait une attention extrêmement aiguë au fonctionnement cognitif, raconte: ‘Gallus Vibius banda si bien son âme à comprendre l'essence et les mouvements de la folie, qu'il emporta son jugement hors de son siège, si qu'oncques puis il ne l'y put remettre; et se pouvoit vanter d'estre devenu fol par sagesse.) (I, 21)

12 Selon Sylvie Dion, ele fait divers se veut toujours le récit d'une quelconque transgression, d'un écart par rapport à une norme (sociale, 
68

sera pas tolérée. Cet avertissement n'est pas si gratuit qu'on pourrait le penser: on sait combien un crime d'un style nouveau et fortement médiatisé tend à engendrer des imitateurs. Il faut éviter la contagion du crime, le déferlement des schèmes criminels dans une mimesis folle.

Les travaux de socio-linguistique de Labov ${ }^{13}$ et Waletzki, tout comme ceux de psychologie cognitive ${ }^{14}$, ont établi que le récit comporte tout naturellement une morale, parfois dénommée, en termes plus laïcs, une évaluation. Même si celle-ci est facultative, elle donne au récit son articulation et en constitue la clé de voûte $^{15}$. Ce fonctionnement est particulièrement apparent dans la fable, où les histoires ne sont retenues que pour la morale, explicite ou implicite, que l'on peut en tirer ${ }^{16}$. Mais si dans la fable le récit est tiré de la moralité, il n'en va évidemment pas de même du fait divers. La fable met à distance la souffrance de la représentation du malheur en l'imputant à des animaux, des végétaux, des choses. En ce sens, elle est éminemment pédagogique, puisqu'elle permet d'affronter la perspective du malheur et de la souffrance par la non-humanisation des personnages qui en sont les victimes. Au contraire, le fait divers monstrueux, qui dramatise notre quotidien, nous confronte avec des événements dont la pensée nous est intolérable et que nous ne pouvons apprivoiser qu'à condition de les fictionnaliser ou de les moraliser. Sur le plan le plus fondamental, le récit n'échappe à l'ordre de la gratuité ou de l'absurde qu'à raison de la dimension morale que l'on peut y trouver - ou y projeter.

Un autre moyen de tenir l'horreur à distance est de la faire passer dans la catégorie de la folie, qui n'est qu'un autre nom de l'anormalité. Foucault nous en a donné un bel exemple avec le dossier constitué au siècle dernier sur le cas de Pierre Rivière,

morale, religieuse, naturelle)*. Voir "Le fait divers comme genre narratif", Imprévue, $\mathrm{n}^{\circ} 2$ (1988), p. 46. Cette caractérisation du fait divers correspond à la catégorie que je range sous le - monstrueux .

13 William Labov, Le parler ordinaire. Vol. 1. La langue dans les ghettos noirs des Etats-Unis, traduit par A. Kihm, Paris, Minuit, 1978.

14 G. Denhière, Il était une fois... Comprébension et souvenir de récits, Lille, Presses universitaires de Lille, 1984.

15 Voir le schéma de Labov, op. cit., p. 306.

16 Voir là-dessus mon ouvrage Apprendre à lire des fables. Une approche sémio-cognitive, Montréal, Le Préambule, coll. "L'Univers des discours", 1989. 
que beaucoup ont voulu tenir pour fou, malgré le caractère prémédité de son acte et son auto-justification fort élaborée ${ }^{17}$. Cette explication par la folie, surtout pour les crimes très violents, n'estelle pas notre dernier recours le plus constant, sans exclure celui de la moralisation - avec laquelle il peut d'ailleurs entrer en conflit, comme on l'a vu lors du massacre de l'École polytechnique? En rejetant l'acte criminel dans la folie, on espère sans doute conjurer la contamination éventuelle par les schèmes qu'il incarne, en les disqualifiant de façon radicale comme s'ils étaient situés hors de cette norme que, par définition, nous croyons toujours incarner ${ }^{18}$ et dans laquelle la lecture du fait divers nous conforte sourdement.

17 Michel Foucault, Moi, Pierre Rivière, ayant égorgé ma mère, ma soeur et mon frère..., Paris, Gallimard/Julliard, 1973.

18 Dans la remarquable étude psychologique qu'il a faite de deux criminels qui avaient commis un crime particulièrement atroce (une famille de quatre personnes: père, mère, fille et fils adolescents), Truman Capote relève une confirmation de cet axiome psychologique là où elle semblerait la moins attendue. Lorsque Dick et Perry apprennent qu'un crime en tout point similaire au leur a été commis exactement un mois plus tard dans une autre ville des États-Unis, ils réagissent en déclarant que l'auteur devait être cinglé, qu'il devait avoir été influencé par le récit de leur crime à eux: "Know what I wouldn't be surprised? If this wasn't done by a lunatic. Some nut that read about what bappened out in Kansas. - (In cold blood, New York, Random House, 1965, p. 200). 